ITEP-PH-6/98

FZ-IKP(TH)-1998-32

\title{
The running BFKL: resolution of Caldwell's puzzle
}

\author{
N.N. Nikolaev ${ }^{\alpha}$ and V. R. Zoller ${ }^{\beta}$ \\ ${ }^{\alpha}$ Institut für Kernphysik, Forschungszentrum Jülich, \\ D-52425 Jülich, Germany \\ E-mail: kph154@ikp301.ikp.kfa-juelich.de \\ ${ }^{\beta}$ Institute for Theoretical and Experimental Physics, \\ Moscow 117218, Russia \\ E-mail: zoller@heron.itep.ru
}

\begin{abstract}
The HERA data on the proton structure function, $F_{2}\left(x, Q^{2}\right)$, at very small $x$ and $Q^{2}$ show the dramatic departure of the logarithmic slope, $\partial F_{2} / \partial \log Q^{2}$, from theoretical predictions based on the DGLAP evolution. We show that the running BFKL approach provides the quantitative explanation for the observed $x$ and/or $Q^{2}$-dependence of $\partial F_{2} / \partial \log Q^{2}$.
\end{abstract}

Caldwell's presentation of the HERA data in terms of the logarithmic derivative $\partial F_{2} / \partial \log Q^{2}$ for the proton structure function $(\mathrm{SF}) F_{2}\left(x, Q^{2}\right)$ exhibits the turn-over of the slope towards small $x$ and/or $Q^{2}$ up to currently attainable $x \sim 10^{-6}$ and $Q^{2} \sim 0.1 \mathrm{GeV}^{2}$ [1, 2]. The DGLAP-evolution [3] with GRV input [4] predicts a steady increase of the derivative

$$
\frac{\partial F_{T}^{D G L A P}}{\partial \log Q^{2}} \propto \alpha_{S}\left(Q^{2}\right) G^{D G L A P}\left(x, Q^{2}\right)
$$

with $1 / x$, due to the growth of the gluon structure function $G^{D G L A P}\left(x, Q^{2}\right)=x g^{D G L A P}\left(x, Q^{2}\right)$. A slight systematic discrepancy of the DGLAP analysis with small- $x$ data on $\partial F_{2} / \partial \log Q^{2}$ was found also in [0].

The turn-over point located at $x \sim 5 \cdot 10^{-4}$ and $Q^{2} \sim 5 \mathrm{GeV}^{2}$, in a commonly believed legitimate pQCD domain. So, the phenomenon occurs on the interface between "soft" and "hard" physics. Its explanation within the color dipole approach is based on two observationsf:

i) specific smallness of the $\log Q^{2}$-derivative of sub-leading terms of the BFKL-Regge expansion for $F_{2}$ at the turn-over point, which is due to the nodal structure of the running BFKL eigen-SF's;

ii) significant contribution to the small- $Q^{2}$ proton SF coming from the non-perturbative

\footnotetext{
${ }^{1}$ The preliminary results have been reported at the DIS'98 Workshop [6]
} 
component of the BFKL pomeron.

The $s$-channel approach to the BFKL equation [7] was developed in terms of the color dipole cross section $\sigma(x, r)$ [8, 9] (hereafter $\vec{r}$ is the color dipole moment). The positive feature of the color dipole picture, to be referred to as the running BFKL approach, is consistent incorporating the two crucial properties of QCD: i) asymptotic freedom (AF), i.e., the running QCD coupling $\alpha_{S}(r)$ and, ii) the finite propagation radius $R_{c}$ of perturbative gluons.

The BFKL equation for the interaction cross section $\sigma(x, r)$ of the color dipole $\vec{r}$ with the target reads

$$
\begin{aligned}
& \frac{\partial \sigma(x, r)}{\partial \log (1 / x)}=\mathcal{K} \otimes \sigma(x, r)= \\
& \frac{3}{8 \pi^{3}} \int d^{2} \vec{\rho}_{1}\left|\overrightarrow{\mathcal{E}}\left(\vec{\rho}_{1}\right)-\overrightarrow{\mathcal{E}}\left(\vec{\rho}_{2}\right)\right|^{2}\left[\sigma\left(x, \rho_{1}\right)+\sigma\left(x, \rho_{2}\right)-\sigma(x, r)\right] .
\end{aligned}
$$

Here the kernel $\mathcal{K}$ is related to the wave function squared of the color-singlet $q \bar{q} g$ state with the Weizsäcker-Williams (WW) soft gluon. The quantity

$$
\overrightarrow{\mathcal{E}}(\vec{\rho})=-g_{S}(\rho) \vec{\nabla}_{\rho} K_{0}\left(\mu_{G} \rho\right)=g_{S}(\rho) \mu_{G} K_{1}\left(\mu_{G} \rho\right) \vec{\rho} / \rho,
$$

where $R_{c}=1 / \mu_{G}$ and $K_{\nu}(x)$ is the modified Bessel function, describes a Yukawa screened transverse chromoelectric field of the relativistic quark and $\left|\overrightarrow{\mathcal{E}}\left(\vec{\rho}_{1}\right)-\overrightarrow{\mathcal{E}}\left(\vec{\rho}_{2}\right)\right|^{2}$ describes the flux (the modulus of the Poynting vector) of WW gluons in the $q \bar{q} g$ state in which $\vec{r}$ is the $\bar{q}-q$ separation and $\vec{\rho}_{1,2}$ are the $q-g$ and $\bar{q}-g$ separations in the two-dimensional impact parameter plane. Our numerical results are for the Yukawa screening radius $R_{c}=0.27 \mathrm{fm}$. The recent fits to the lattice QCD data on the field strength correlators suggest similar $R_{c}$ [10].

The asymptotic freedom of QCD uniquely prescribes the chromoelectric field be computed with the running QCD charge $g_{S}(r)=\sqrt{4 \pi \alpha_{S}(r)}$ taken at the shortest relevant distance, $R_{i}=\min \left\{r, \rho_{i}\right\}$ in the $q \bar{q} g$ system. Although, the so introduced running coupling does not necessarily exhaust all NLO effects, it correctly describes the crucial enhancement of long distance, and suppression of short distance, effects by AF.

The properties of the running color dipole BFKL equation responsible for the observed $Q^{2}$ dependence of $\partial F_{2} / \partial \log Q^{2}$ are as follows [11, 12]. The spectrum of the running BFKL equation is a series of moving poles in the complex $j$-plane with eigen-functions

$$
\sigma_{n}(x, r)=\sigma_{n}(r) \exp \left[\Delta_{n} \log (1 / x)\right]
$$

being a solution of

$$
\mathcal{K} \otimes \sigma_{n}=\Delta_{n} \sigma_{n}(r)
$$

The leading eigen-function $\sigma_{0}(r)$ is node free. The sub-leading $\sigma_{n}(r)$ has $n$ nodes. The intercepts $\Delta_{n}$ closely, to better than $10 \%$, follow the law $\Delta_{n}=\Delta_{0} /(n+1)$ suggested earlier by Lipatov 13. The intercept of the leading pole trajectory, with the above specific choice of $R_{c}$, is $\Delta_{0} \equiv \Delta_{\mathbb{I P}}=0.4$. The sub-leading eigen-functions $\sigma_{n}$ [11, 12] are very close to Lipatov's quasi-classical solutions [13] for $n \gg 1$. For our specific choice of the infrared regulator, $R_{c}$, the node of $\sigma_{1}(r)$ is located at $r=r_{1} \simeq 0.05-0.06 \mathrm{fm}$, for larger $n$ the first node moves to a somewhat larger $r \sim 0.1 \mathrm{fm}$. 
The color dipole factorization [14] in conjunction with the explicit form of the $q \bar{q}$ lightcone wave function, $\Psi_{q q}(z, r)$, relates the dipole cross sections $\sigma_{n}(r)$ with the eigen-SF, $f_{n}\left(Q^{2}\right)$

$$
f_{n}\left(Q^{2}\right)=\frac{Q^{2}}{4 \pi \alpha_{e m}} \sum_{q=u, d, c, s} \int_{0}^{1} d z \int d^{2} \vec{r}\left|\Psi_{q q}(z, r)\right|^{2} \sigma_{n}(r),
$$

The BFKL-Regge expansion

$$
\sigma(x, r)=\sigma_{0}(r)\left(x_{0} / x\right)^{\Delta_{0}}+\sigma_{1}(r)\left(x_{0} / x\right)^{\Delta_{1}}+\sigma_{2}(r)\left(x_{0} / x\right)^{\Delta_{2}}+\ldots .
$$

gives the BFKL-Regge expansion for the SF

$$
F_{2}\left(x, Q^{2}\right)=\sum_{n} f_{n}\left(Q^{2}\right)\left(x_{0} / x\right)^{\Delta_{n}} .
$$

The remarkable finding of 115, 11, 12 is a good description of the HERA data on the proton SF starting with the Born two-gluon cross section $\sigma_{B}(r)$ as a boundary condition for the running BFKL equation (2) at $x_{0}=0.03$. With such a boundary condition, which could well be excessively restrictive, the expansion (7) fixes uniquely the normalization of the eigen-FS's.

The Bjorken variable $x=Q^{2} / 2 m_{p} \nu$ is commonly being used for the presentation of the experimental data even at $Q^{2} \lesssim m_{\rho}^{2}$, way beyond the kinematical region $Q^{2} \gg m_{\rho}^{2}$ it has originally been devised for. At small $Q^{2}$, the relevant Regge parameter is $2 m_{p} \nu /\left(Q^{2}+m_{\rho}^{2}\right)$ rather than the $1 / x$. Consequently, in the small- $Q^{2}$ region the Regge parameter $x_{0} / x$ in eqs.(7) and (8) must be substituted by $\left(x_{0} / x\right)\left(1+m_{\rho}^{2} / Q^{2}\right)$.

One more remark on kinematics is in order. The BFKL-Regge expansion (8) holds at small $x \lesssim 10^{-2}$. In order to model the sea contribution at larger $x$ we multiply (8) by the familiar factor $(1-x)^{m}$, with $m=5$. This factor does not affect the diffraction region but strongly suppresses production of gluons with $x \gtrsim 0.1$.

In applications it is convenient to work with $f_{n}\left(Q^{2}\right)$ represented in an analytical form. For the leading singularity we have

$$
f_{0}\left(Q^{2}\right)=a_{0} \frac{R_{0}^{2} Q^{2}}{1+R_{0}^{2} Q^{2}}\left[1+c_{0} \log \left(1+r_{0}^{2} Q^{2}\right)\right]^{\gamma_{0}},
$$

which has the large- $Q^{2}$ asymptotics [16, 9]

$$
f_{0}\left(Q^{2}\right) \propto\left[\alpha_{s}\left(Q^{2}\right)\right]^{-\gamma_{0}}, \quad \gamma_{0}=\frac{4}{3 \Delta_{0}}
$$

For $n \geq 1$ the functions $f_{n}\left(Q^{2}\right)$ can be approximated by

$$
f_{n}\left(Q^{2}\right)=a_{n} f_{0}\left(Q^{2}\right) \frac{1+R_{0}^{2} Q^{2}}{1+R_{n}^{2} Q^{2}} \prod_{i=1}^{n_{\max }}\left(1-\frac{z}{z_{n}^{(i)}}\right)
$$

where

$$
z=\left[1+c_{n} \log \left(1+r_{n}^{2} Q^{2}\right)\right]^{\gamma_{n}}-1, \quad \gamma_{n}=\gamma_{0} \delta_{n}
$$

and $n_{\max }=\min \{n, 2\}$.

Since the relevant variable is a power of the inverse gauge coupling the nodes of $f_{n}\left(Q^{2}\right)$ are spaced by 2-3 orders of magnitude in $Q^{2}$-scale and only the first two of them are in 
the accessible range of $Q^{2}$ [11, [12]. The first nodes of sub-leading $f_{n}\left(Q^{2}\right)$ are located at $Q^{2} \sim 20-60 \mathrm{GeV}^{2}$, the second nodes of $f_{2}\left(Q^{2}\right)$ and $f_{3}\left(Q^{2}\right)$ are at $Q^{2} \simeq 5 \cdot 10^{3} \mathrm{GeV}^{2}$ and $Q^{2} \simeq 2 \cdot 10^{4} \mathrm{GeV}^{2}$, respectively. The parameterization tuned to reproduce the numerical results for $f_{n}\left(Q^{2}\right)$ at $Q^{2} \lesssim 10^{5} \mathrm{GeV}^{2}$ is given by eq.(11). For $n=3$ we take a simplified form with only two first nodes, because the third node of $f_{3}\left(Q^{2}\right)$ is at $\sim 2 \cdot 10^{7} \mathrm{GeV}^{2}$, way beyond the reach of accelerator experiments at small $x$. The found parameters are listed in the Table.

\begin{tabular}{|l|l|l|l|l|l|l|l|l|}
\hline$n$ & $a_{n}$ & $c_{n}$ & $r_{n}^{2}, \mathrm{GeV}^{-2}$ & $R_{n}^{2}, \mathrm{GeV}^{-2}$ & $z_{n}^{(1)}$ & $z_{n}^{(2)}$ & $\delta_{n}$ & $\Delta_{n}$ \\
\hline 0 & 0.0232 & 0.3261 & 1.1204 & 2.6018 & & & & 0.40 \\
\hline 1 & 0.279 & 0.1113 & 0.8755 & 3.4648 & 2.4773 & & 1.0915 & 0.220 \\
\hline 2 & 0.195 & 0.0833 & 1.5682 & 3.4824 & 1.7706 & 12.991 & 1.2450 & 0.148 \\
\hline 3 & 0.471 & 0.0653 & 3.9567 & 2.7756 & 1.4963 & 6.9160 & 1.2284 & 0.111 \\
\hline
\end{tabular}

Asymptotically, at $1 / x \rightarrow \infty$, the expansion (8) is dominated by the term $f_{0}\left(Q^{2}\right)\left(x_{0} / x\right)^{\Delta_{0}}$. At moderately small $x$ the sub-leading terms are equally important since $\Delta_{n} \sim 1 / n$. However, as it has been pointed out in [11, 12], for $Q^{2} \lesssim 10^{4} \mathrm{GeV}^{2}$ all $f_{n}\left(Q^{2}\right)$ with $n \geq 3$ are very close in shape to each other. Then we arrive at the truncated expansion

$$
F_{2}\left(x, Q^{2}\right)=\sum_{n=0}^{3} f_{n}\left(Q^{2}\right)\left(x_{0} / x\right)^{\Delta_{n}}+F_{2}^{s o f t}\left(Q^{2}\right)+F_{2}^{v a l}\left(x, Q^{2}\right)
$$

where the term $f_{3}\left(Q^{2}\right)\left(x_{0} / x\right)^{\Delta_{3}}$ with the properly adjusted weight factor, $a_{3}$, stands for all terms with $n \geq 3$. The addition of this "background" term in eq.(13) improves significantly the agreement with data for large $Q^{2}$ thus expanding the applicability region of eq. (13) over the whole small- $x$ kinematical domain of HERA.

The need for a soft pomeron contribution $F_{2}^{\text {soft }}$ in addition to the perturbative gBFKL SF's described previously is brought about by phenomenological considerations. A viable gBFKL phenomenology of the rising component of the proton structure function over the whole range of $Q^{2}$ studied at HERA (real photo-absorption included) is obtained if one starts with the Born dipole cross section $\sigma_{B}(r)$ as a boundary condition for the gBFKL evolution at $x_{0}=0.03$ [17, 12]. However, such a purely perturbative input, $\sigma_{B}(r)$, with $R_{c}=0.27 \mathrm{fm}$ strongly underestimates the cross sections of soft processes and the proton SF at moderate $Q^{2} \sim 1 \mathrm{GeV}^{2}$. Therefore, at $r \gtrsim R_{c}$, the above described perturbative gBFKL dipole cross section $\sigma_{p t}(x, r)$, must be complemented by the contribution from the non-perturbative soft pomeron, $\sigma_{n p t}(x, r)$. In terms of the relationship [17] between $\sigma(x, r)$ and the gluon structure function of the proton, $G\left(x, Q^{2}\right)$, the non-perturbative dipole cross section $\sigma_{n p t}(r)$ at $r \gtrsim R_{c}$ must be associated with soft non-perturbative gluons in the conventional $G\left(x, Q^{2}\right)$. The contribution to $G\left(x, Q^{2}\right)$ from the non-perturbative transverse momenta $k^{2} \lesssim Q_{0}^{2} \sim m_{\rho}^{2}$ persists at all $Q^{2}$ and must not be subjected to the DGLAP evolution.

Because the BFKL rise of $\sigma(x, r)$ is due to production of $s$-channel perturbative gluons, which does not contribute to $\sigma_{n p t}(r)$ in [17, 12] we argued that to a first approximation one must consider the energy independent $\sigma_{n p t}(r)$ and additivity of scattering amplitudes from both the hard BFKL and soft non-perturbative mechanisms. For recent suggestions to identify our $\sigma_{n p t}(r)$ with the soft pomeron of the two-pomeron picture see [18, 19]. In the models of soft scattering via polarization of the non-perturbative QCD vacuum [20, 21], $\sigma_{n p t}(r)$ is interpreted in terms of the non-perturbative gluon distributions. 
To our opinion, the recently encountered troubles with the small- $Q^{2}$ extrapolations of DGLAP evolution [5] and the failure of DGLAP fits in the Caldwell plot [1], [2] are due to illegitimate enforcing the DGLAP evolution upon the non-perturbative glue.

The non-perturbative term $F_{2}^{\text {soft }}\left(Q^{2}\right)$ in eq.(13) calculated from eq.(6) with $\sigma=\sigma_{n p t}(r)$ from 22 can be parameterized as follows

$$
F_{2}^{\text {soft }}\left(Q^{2}\right)=b \frac{R^{2} Q^{2}}{1+R^{2} Q^{2}}\left[1+c \log \left(1+r^{2} Q^{2}\right)\right]
$$

where $b=0.1077, c=0.0673, R^{2}=6.6447 \mathrm{GeV}^{-2}$ and $r^{2}=7.0332 \mathrm{GeV}^{-2}$. So, its $\log Q^{2}$ derivative levels off at very small $Q^{2} \sim 0.15 \mathrm{GeV}^{2}$ and does not contribute to the observed growth of $\partial F_{2} / \partial \log Q^{2}$.

In Fig. 1 we confront our estimates to both the HERA data and the fixed target data. In (2) the logarithmic slope, $\partial F_{2} / \partial \log Q^{2}$, is derived from data by fitting $F_{2}=a+b \log Q^{2}$ in bins of fixed $x$. The average value of $Q^{2},\left\langle Q^{2}\right\rangle$, in each $x$-bin is derived from the $F_{2}$ weighted mean value of $\log Q^{2}$ in that bin.

As we have noticed above, at moderately small $x \sim 10^{-2}-10^{-3}$ the contribution of the sub-leading poles to $F_{2}\left(x, Q^{2}\right)$ is still substantial (the relative weight factors, $a_{n}$, with $n \geq 1$ are presented in the table), but toward the region of $x \sim 10^{-6}$ the leading pole contribution starts to prevail.At small $Q^{2}$ the ratio of $\log Q^{2}$-derivatives, $r_{n}=f_{n}^{\prime} / f_{0}^{\prime}$, can be estimated as

$$
r_{n} \simeq a_{n} \frac{\Lambda_{0}^{2}}{\Lambda_{n}^{2}}\left(\frac{x}{x_{0}}\right)^{\Delta_{0}-\Delta_{n}}
$$

where

$$
\Lambda_{n}^{2} \simeq 1 /\left(R_{n}^{2}-c_{0} \gamma_{0} r_{0}^{2}\right)
$$

Because the sub-leading SF's, $f_{n}\left(Q^{2}\right)$, have node at $Q^{2} \sim 20-60 \mathrm{GeV}^{2}$ 11, 12, their contribution to the slope $\partial F_{2} / \partial \log Q^{2}$ vanishes at $Q^{2} \sim 5-10 \mathrm{GeV}^{2}$, which is very close to the turn-over point in the HERA data. Hence $\partial F_{2} / \partial \log Q^{2}$ at small $Q^{2}$ follows closely $\partial f_{0} / \partial \log Q^{2}$. From (9) it follows that at small $Q^{2}, f_{0}\left(Q^{2}\right)$ behaves like $\sim Q^{2} /\left(\Lambda_{0}^{2}+Q^{2}\right)$ with $\Lambda_{0}^{2} \simeq 0.72 \mathrm{GeV}^{2}$ coming from (16). Therefore, $\partial F_{2} / \partial \log Q^{2}$ rises with $Q^{2}$ up to $Q^{2} \sim 1 \mathrm{GeV}^{2}$ then levels off. Only at large $Q^{2}$, when the sub-leading terms enter the game, $\partial F_{2} / \partial \log Q^{2}$ decreases and even becomes negative valued at large $x$. Our estimates shown in Fig.1a are in good agreement with HERA data [2]. The curves are somewhat wiggly because the $x-\left\langle Q^{2}\right\rangle$ correlation of the experimental data is non-monotonous one.

In Fig.1b we compare our predictions with the fixed target data [1]. Variation of the slope in this case is less pronounced since the starting value of $\left\langle Q^{2}\right\rangle$ is $\left\langle Q^{2}\right\rangle \simeq 0.54 \mathrm{GeV}^{2}$ at $x \simeq 10^{-3}$ (compare with $Q^{2}=0.12 \mathrm{GeV}^{2}$ at $x=2.1 \cdot 10^{-6}$ at HERA). It can easily be seen that the derivative $\partial f_{0} / \partial \log Q^{2}$ at such $Q^{2}$ is a rather slow function of $Q^{2}$. The agreement of our estimates with the fixed target data is quite reasonable, though there is a systematic

discrepancy at small $x$. We recall that there is a certain mismatch between the E665 and H1/ZEUS data on $F_{2}\left(x, Q^{2}\right)$ in the close $\left(x, Q^{2}\right)$ bins (see Fig.2a and Fig.2b).

\section{References}

[1] A. Caldwell, DESY Theory Workshop, DESY, October 1997. 
[2] ZEUS Collaboration, J. Breitweg et al., ZEUS results on the measurement and phenomenology of $F_{2}$ at low $x$ and low $Q^{2}$, Report No. DESY-98-162

[3] V.N. Gribov and L.N. Lipatov, Sov. J. Nucl. Phys. 15 (1972) 438; G. Altarelli and G. Parisi, Nucl. Phys. B126 (1977) 298; Yu.L. Dokshitzer, Sov. Phys. JETP 46 (1977) 641.

[4] M. Glück, E. Reya and A. Vogt, Z. Phys. C67 (1995) 433.

[5] A.D. Martin, R.G. Roberts, W.J. Stirling and R.S. Thorne, Parton distributions: a new global analysis, Report No.DTP/98/10; RAL-TR-98-029

[6] V.R. Zoller, in Proc. 6th Int. Workshop on DIS and QCD (DIS 98), Brussels, 1998. Report No.FZ-IKP(TH)-1998-5

[7] E.A.Kuraev, L.N.Lipatov and V.S.Fadin, Sov.Phys. JETP 44 (1976) 443; 45 (1977) 199; Ya.Ya.Balitskii and L.N.Lipatov, Sov. J. Nucl. Phys. 28 (1978) 822.

[8] N.N. Nikolaev, B.G. Zakharov, V.R. Zoller, JETP Letters 59 (1994) 8.

[9] N.N. Nikolaev, B.G. Zakharov, V.R. Zoller , JETP 105 (1994) 1498.

[10] E. Meggiolaro, Field strength correlators in QCD: new fits to the lattice data, Report No. HD-THEP-98-34

[11] V.R. Zoller in Proc. 5th Int. Workshop on DIS and QCD (DIS 97), Chicago, IL, 1997.

[12] N.N. Nikolaev, B.G. Zakharov, V.R. Zoller,JETP Letters 66 (1997) 138.

[13] L.N. Lipatov, Sov. Phys. JETP 63 (1986) 904.

[14] N.N. Nikolaev and B.G. Zakharov, Z. Phys. C49 (1991) 607.

[15] N.N. Nikolaev and B.G. Zakharov Phys. Lett. B333 (1994) 250.

[16] N.N. Nikolaev, B.G. Zakharov, Phys. Lett. B327 (1994) 157.

[17] N.N.Nikolaev and B.G.Zakharov, Phys. Lett. B327 (1994) 149.

[18] A. Donnachie and P.V. Landshoff, Phys.Lett. B437 (1998) 408.

[19] K. Golec-Biernat and M. Wüsthoff, DTP-98-50, Jul 1998.

[20] P.V.Landshoff and O.Nachtmann, Z. Phys. C35 (1987) 405.

[21] H.G.Dosch, T.Gousset, G.Kulzinger et al., Phys. Rev. D55 (1997) 2602.

[22] J. Nemchik, N.N. Nikolaev, E. Predazzi, B.G. Zakharov and V.R. Zoller, JETP 86 (1998) 1054. 


\section{Figure Captions}

Fig.1 Caldwell's plot of $\partial F_{2} / \partial \log Q^{2}$ for the ZEUS data [2] (Fig. 1a) and fixed target data [1] (Fig. 1b). Our predictions (BFKL-Regge) are shown by the solid lines. Shown by the dashed lines is the leading BFKL pole approximation (LPA).

Fig.2 Description of the H1, ZEUS and E665 $F_{2}\left(x, Q^{2}\right)$ data by the BFKL-Regge expansion (8): the large- $Q^{2}$ data $\left(Q^{2}=3.5,12,25,65,120\right.$ and $\left.200 \mathrm{GeV}^{2}\right)$ are shown in Fig.2a, the small- $Q^{2}$ data $\left(Q^{2}=0.11,0.20,0.40,0.65,0.85\right.$ and $\left.1.2 \mathrm{GeV}^{2}\right)$ are in Fig.2b. For display purposes we have multiplied $F_{2}$ by the numbers shown in brackets. 


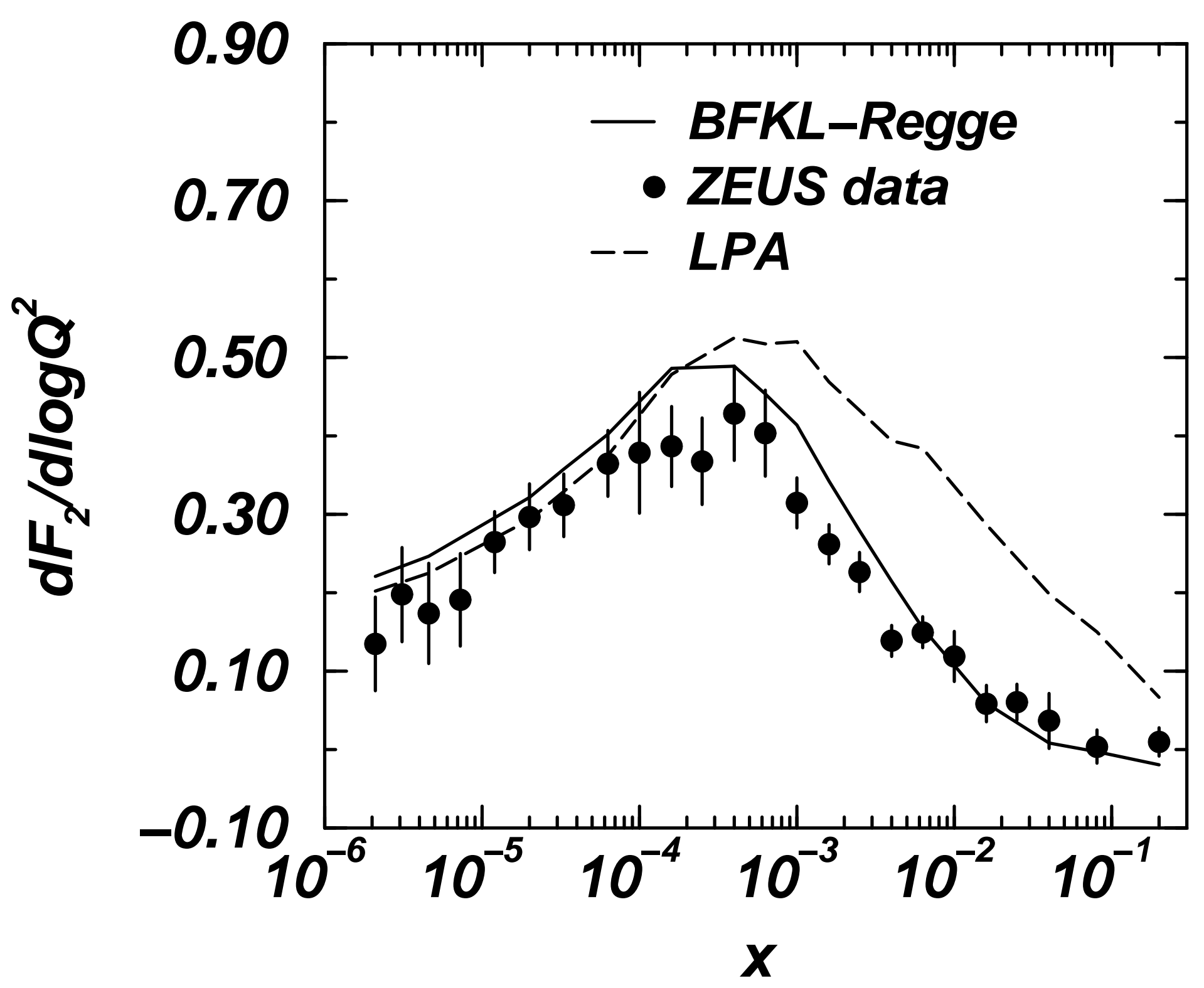




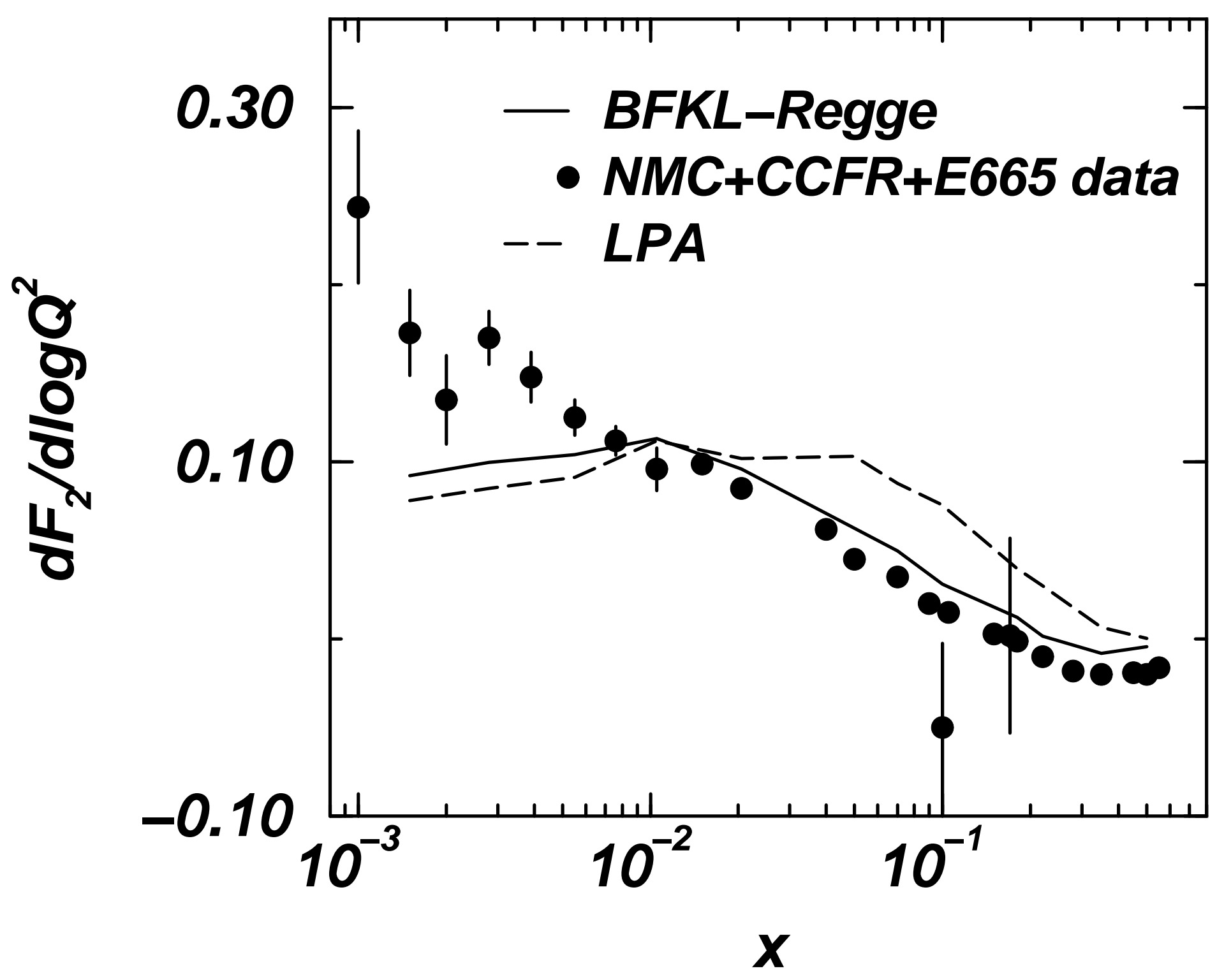




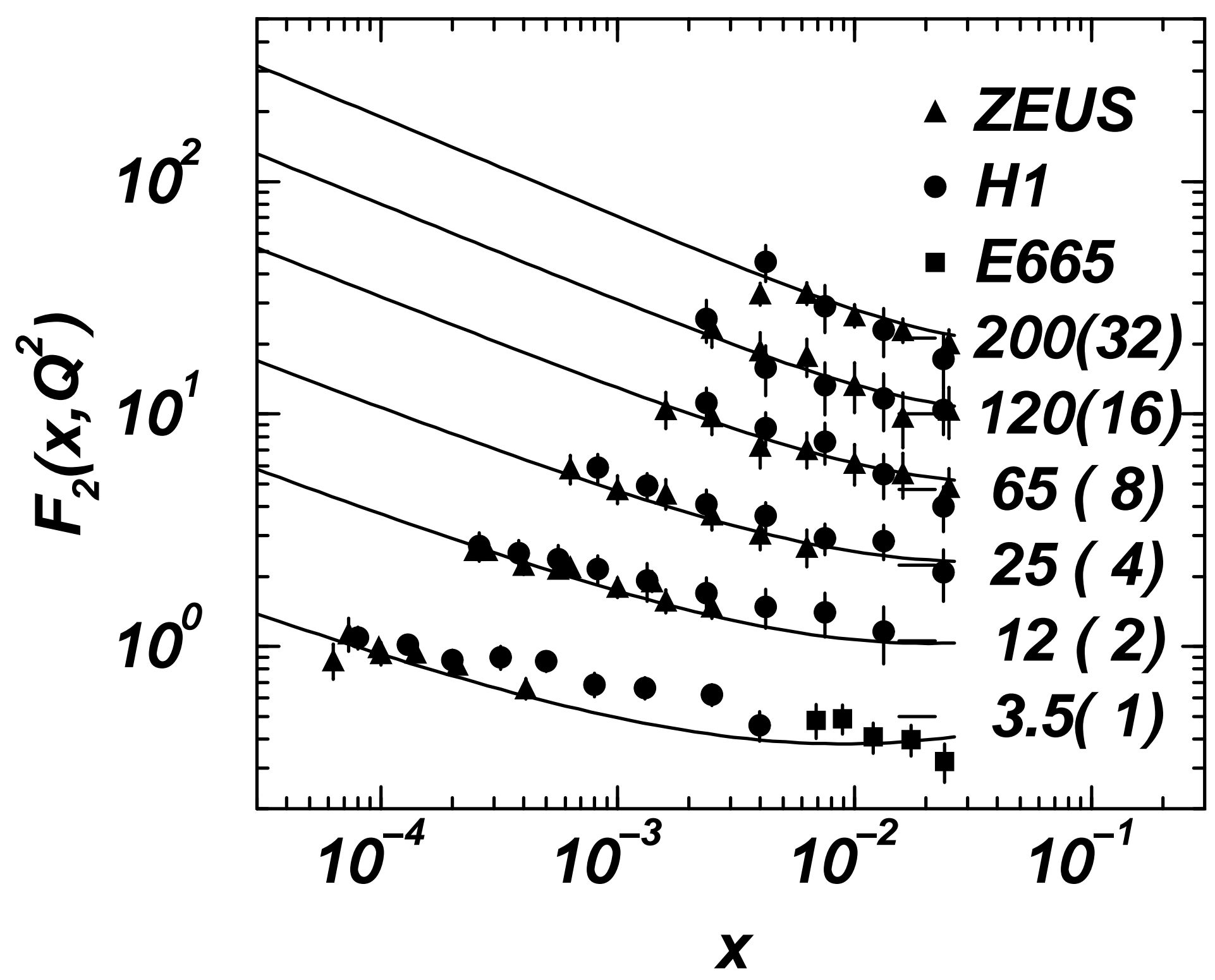




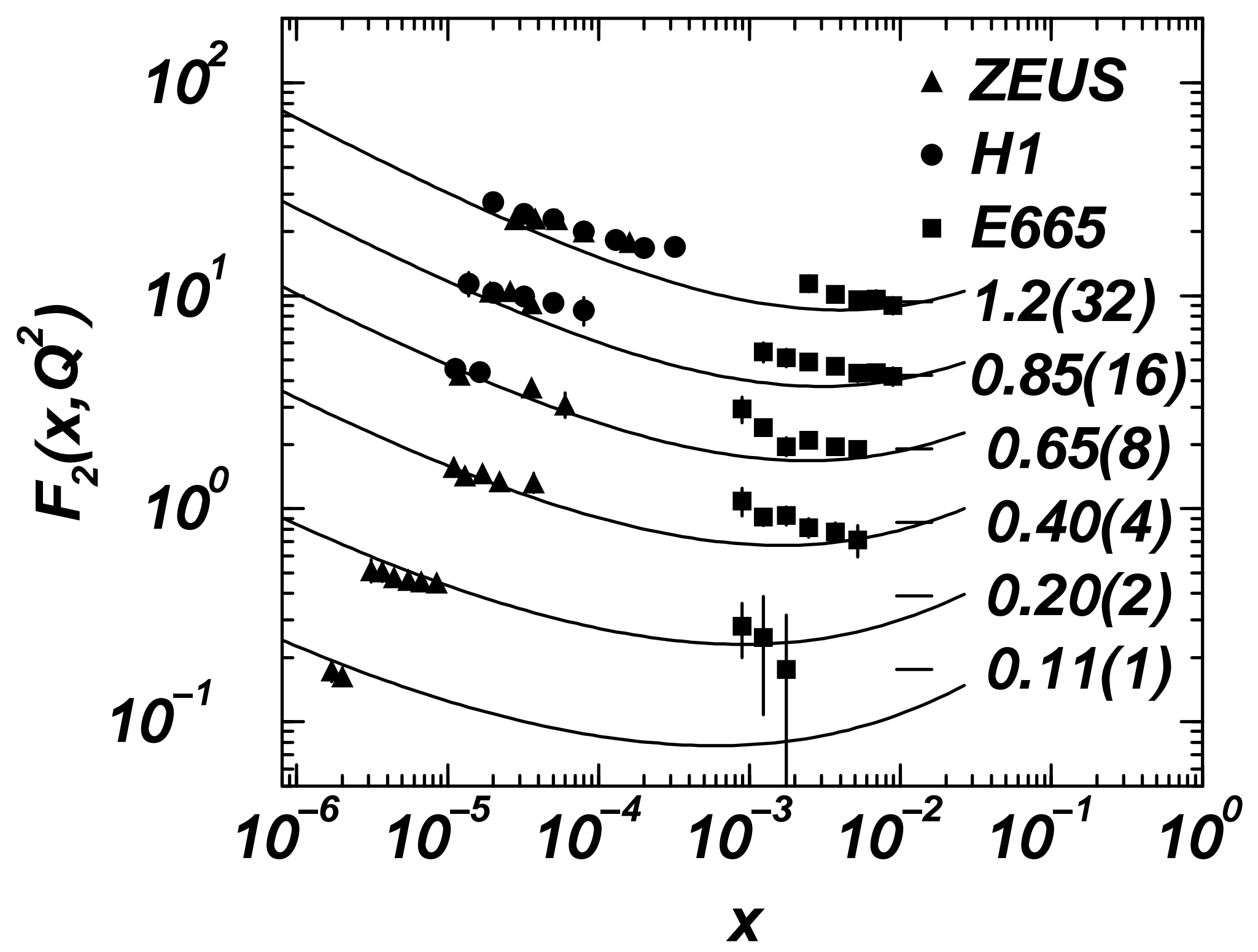

